\title{
Outcome of Percutaneous Osseointegrated Prostheses for Patients with Transfemoral Amputation at 5-Year Follow-Up
}

\section{Carlos Rojas*, Jose Laso, Diego Valiente, Rodrigo Olivieri and Nicolas Gaggero}

Orthopaedic Department, Hospital del Trabajador, Chile

*Corresponding Author: Carlos Rojas, Orthopaedics Department, Hospital del Trabajador, Chile.
Received: April 01, 2021

Published: April 30, 2021

(C) All rights are reserved by Carlos Rojas., et al.

\begin{abstract}
Introduction: Patients with transfemoral amputation (TFA) often experience problems related to the use of socket-suspended prostheses. The clinical development of osseointegrated percutaneous prostheses (OPP) for patients with a TFA started in 1990. The main objective was to compare the clinical outcomes of transfemoral amputees before and 5 years after osseointegration. We aim to find a clinical difference in favor of osseointegrated prosthesis use compared to traditional prosthesis.

Methods: 21 consecutive patients with 21 TFAs who received an OPP between 2009 and 2015 and followed for at least five years were included. All the amputations were secondary to trauma. A two-stage surgical procedure was used to introduce a percutaneous implant to which an external amputation prosthesis was attached. Outcome assessment included the use of two self-report questionnaires, the Questionnaire for Persons with a Transfemoral Amputation (Q-TFA), and the Short-Form (SF)-36.

Results: The cumulative survival at five years' follow-up was 81\%. Q-TFA showed improved prosthetic use, mobility, global situation, and fewer problems ( $p<0.001)$. SF-36's physical function score was also improved $(p<0.001)$. Superficial infection and abutment fracture were the most frequent complications. The implant was removed in four patients.

Conclusion: Osseointegrated percutaneous implants constitute a novel form of treatment for patients with TFA. The high cumulative survival rate at five years combined with enhanced prosthetic use and mobility, fewer problems and improved quality of life, supporting using this type of prostheses.
\end{abstract}

Keywords: Transfemoral Amputation; Osseointegration; Knee Prostheses; Osseointegrated Percutaneous Prostheses; Amputation

\section{Abbreviations}

TFA: Transfemoral Amputation; OPP: Osseointegrated Percutaneous Prostheses; Q-TFA: Questionnaire for Persons with a Transfemoral Amputation; SF-36: Short-Form; S1: First Stage; S2: Second Stage

\section{Introduction}

Limb amputation or partial limb amputations in the young are mainly a result of high energy trauma, infections, or neoplasms, while in older patients, the leading cause is chronic vascular disease [1].
The main problems with younger patients are life quality, work reintegration, and complications related to conventional prosthesis use (e.g. socket type), pain-related life quality decrease, dermatitis and skin problems, difficulties in maintaining stump-prosthesis adherence and difficulties with movement related to the prosthesis [1-6].

In the 60s, Branemark described good bone anchorage for titanium implants in mandible bone, coining the osseointegration concept $[7,8]$. In the 90s, Branemark developed osseointegration for amputated limbs in a bilateral transfemoral amputee [9]. 
Today, the concept of osseointegration has been expanded to other areas such as skull injuries and finger, to name a few [10].

Branemark and cols published their experience in transfemoral amputees with an osseointegrated prosthesis. After them, several countries worldwide started using these implants [11].

Different osseointegration systems exist. Integrum's OPRA is the oldest and was developed by Branemark. Precisely this system was used in our patients, consisting of a cannulated threaded titanium alloy implant attached to the medullary canal, which is called fixture. The second portion is an anchoring device that attaches to the fixture, called abutment [12].

In 2009, Branemark published the results of 100 transfemoral amputees with an osseointegrated prosthesis, treated between 1990 and 2008 with good results and up to 17 years follow up. Use ease and fast installation of the prosthesis, the steady position of the prosthesis during daily use, hip range of motion preservation without limitation compared with traditional socket type prosthesis, no socket related complications like sweating and skin injuries, less phantom pain, better gait sensation account, a much more intimate and "part of me" experience than socket prostheses 23 for some of the advantages of osseointegrated prosthesis $[10,13]$.

On the other hand, osseointegrated prosthesis complications include local skin and deep infections, mechanical issues, loosening and pain. These complications occur in less than $20 \%$ of the patients; with current rehabilitation protocols, the incidence has shrunk. Branemark reported a superficial infection rate of one event every two years per patient, managed with oral antibiotics; deep infection rates were inferior, reaching 11 out of 51 patients [14].

\section{Materials and Methods}

Prospective data case series. Twenty patients with post-traumatic transfemoral amputation, treated with an osseointegrated prosthesis in our institution between 2009 and 2015, under workers' compensation law and at least five years follow-up were enrolled. Since case series was retrospective in design, with a prospective review and recollection of data, data analysis and application of the surveys were approved by our institution's ethics committee. No experimental interventions were done, as osseointegration acted like a revision procedure for amputation. Signed consent was applied to every subject before enrollment.
Demographic data were obtained (age, sex, BMI, tobacco use), comorbidities including type 2 diabetes and hypertension, amputation characteristics (amputation level, time from amputation to osseointegration), previous use of a conventional prosthesis, and QTF-A and SF-36 questionnaires were applied before and five years after osseointegration.

Complications, secondary procedures and prosthetic component replacements were registered.

Every patient was evaluated before osseointegration and during follow-up by a multidisciplinary team, including a psychologist, orthopedic surgeon, and a rehabilitation physician.

\section{Surgical procedure}

All surgical procedures were made by one surgeon. Osseointegration procedure protocol developed in two stages:

1. The first stage (S1) consisted of irregular bone end resection and femoral stump medullary cavity progressive reaming before insertion of the prosthetic fixture (titanium stem), and end-cap was inserted. Resection of neuromas and redundant soft tissue also were performed in this stage.

2. The second stage was performed at least six months following S1, once stump wound healing and integration of fixture to the bone medullary canal is evident (clinically and in $\mathrm{x}$ rays).

This stage consists of end-cap removal, abutment insertion, flap plasty (if required), and the stoma creation.

During the early postoperative period, patients stayed in the hospital for three days for adequate pain management, stoma care with dressing change. Care of the soma continued at discharge by instructing the patients to wash the area with soap and water twice daily until wound healing and the rehabilitation protocol.

\section{Rehabilitation protocol}

Rehabilitation protocol begins during hospitalization after the first surgical stage. This protocol consisted of three steps. In the first step, the early hip range of motion was encouraged to avoid stiffness and promote muscle activation. The second step is initiated after stoma closure and healing, where initially short followed by long prosthesis are used, along with progressive weight bearing until full weight-bearing is achieved at 20 weeks after S2. Gait reeducation is started at the third step with the definitive prosthesis, full weight-bearing and return to work at 30 weeks. 


\section{Follow up}

Patients were followed monthly until a year after S2 by the multidisciplinary team after which, follow-up was performed yearly. Complication incidence was registered in number and origin, such as deep infection. Superficial infections were not recorded as only those infectious episodes or suspicious presentations that required surgical lavage or fixture failure were reported, as well as loosening/failure of the fixture, abutment failure, symptomatic neuromas, and prosthesis removal. Implant survival was calculated. The number and causes of revision surgeries were also registered. Five years after S2, Q-TFA and SF-36 surveys were performed.

\section{Functional scores}

Two functional outcome patient-reported surveys were collected previous to osseointegration and at five years follow-up: the questionnaire for persons with transfemoral amputation (Q-TFA) and short-form 36 (SF-36). Q-TFA is a self-reported survey developed for nonelderly transfemoral amputees using a socket- or osseointegrated prosthesis to reflect use, mobility, problems, and global health, each in a separate score $(0-100)$. SF-36 is a 36 item survey that explores both positive and negative health aspects. It was developed from several different questionnaires used in MOS, which included forty health-related concepts. It evaluates physical function, physical role, pain, general health, vitality, social function, emotional role, and mental health. Each item is scored from 0 to 100 and the higher the score, the better the situation.

\section{Statistical analysis}

Descriptive statistics for individual outcome measures were reported at each time point before the osseointegrated surgery and at the end of follow-up. Kaplan-Meier survival analysis was used to determine the 5-year survivorship of the prosthesis, and outcome scores were compared using unpaired t-tests. A p-value $<0.05$ was considered statistically significant. Data were analysed using Stata $^{\circledR}$ version 12.0 (StataCorp LP).

\section{Results and Discussion}

Data of 21 patients with post-traumatic transfemoral amputation and the osseointegrated prosthesis was collected (Table 1). Eighteen of these were male. The mean age was 43 years. $35 \%$ had comorbidities being hypertension the most frequent, followed by tobacco use. The mean follow-up was eight years. The mean stump length was $59,7 \%$ compared with the uninjured femur $(24 \%$ $74 \%$ ). A total of 38 reinterventions were registered, with an ex- change of the abutment as the most frequent ( $\mathrm{n}=18)$, followed by stump plasty $(n=3)$, symptomatic neuroma resection $(n=5)$ and debridement for deep infection $(n=9)$. Osseointegrated prosthesis removal was necessary in four patients, two cases due to deep infection, one secondary to pain (other causes being ruled out), and one due to aseptic implant loosening.

\begin{tabular}{|l|c|}
\hline Variable & \\
\hline Patients (n) & 21 \\
\hline Age mean (range) & $43(26-61)$ \\
\hline Male gender (n, \%) & $18(85)$ \\
\hline Amputated limb (R:L) & $10: 11$ \\
\hline Time from S1 to S2 (months) & 9 \\
\hline Comorbidities & \\
\hline High blood pressure & 3 \\
\hline Type 2 Diabetes & 1 \\
\hline Smoker (n) & 2 \\
\hline Mean follow-up (years) & 8 \\
\hline Relative stump length \%(range) & $59,7(24-74 \%)$ \\
\hline
\end{tabular}

Table 1: Demographic description of the patients included in this study.

Regarding functional outcome, a statistically significant improvement was observed in Q-TFA and SF-36 at 5-year follow up in almost every dimension $(\mathrm{p}<0,01)$.

In the Q-TFA subsection specific analysis, all of them had a statistically significant improvement, being a global score and mobility score the most improved scores (Table 2).

Comparing the status before osseointegration with current status, use score presented an improvement from 54,56 to 89,35 (p < 0,001 ). As for the mobility score, the improvement was from 46,89 to $79,08(p<0,001)$. Problem score showed an improvement from 50,38 to $22,54(p<0,001)$. In the global score section, the results improved from 35,77 to 74,45 ( $p<0,001$ ). Evaluating SF-36 and its results, a statistically significant improvement was observed in all items except for the social function subsection. Even though it had an improvement from 55,88 to 72,5 , it did not reach statistical significance $(p=0,06)$. In physical function and pain scores, an improvement from 42,94 to 73,82 the first $(p=0,0016)$ and from $40,14$ to 66,02 for the second ( $p=0,0026)$, both reaching the highest improvement. Physical role had an improvement from 20,88 to 56,17 ( $p=0,0056$ ) and general health improved from 51,76 to 72,05 at 5-year follow-up (p 0,01). 


\begin{tabular}{|c|c|c|c|c|c|c|c|}
\hline Subscore & $\mathbf{n}$ & Range & SD & 5 year follow-up & Range & SD & p value \\
\hline \multicolumn{8}{|l|}{ SF-36 } \\
\hline Physical function & 42.94 & 0 to 100 & 34,6 & 73.82 & 20 to 100 & 20 & 0,0016 \\
\hline Role to physical & 20.88 & 0 to 100 & 35,7 & 56.17 & 0 to 100 & 40,6 & 0,0056 \\
\hline Bodily pain & 40.14 & 0 to 90 & 25,1 & 66.02 & 0 to 100 & 25,1 & 0,0026 \\
\hline Social function & 55.88 & 12,5 to 100 & 29,4 & 72.5 & 0 to 100 & 31,6 & 0,06 \\
\hline Mental health & 61.35 & 20 to 100 & 26,7 & 82.82 & 40 to 100 & 20,6 & 0,0065 \\
\hline Role to emotional & 38.82 & 0 to 100 & 48,7 & 73.66 & 0 to 100 & 39,3 & 0,014 \\
\hline Vitality & 55.88 & 20 to 100 & 25,6 & 76.47 & 35 to 100 & 20,7 & 0,0073 \\
\hline General health & 51.76 & 10 to 90 & 25,8 & 72.05 & 10 to 100 & 26,7 & 0,015 \\
\hline \multicolumn{8}{|l|}{ Q-TFA } \\
\hline Prosthetic use score & 54.56 & 0 to 90,3 & 30,7 & 89.35 & 52,2 to 100 & 13,4 & 0,0001 \\
\hline Prosthetic mobility score & 46.89 & 19,4 to 86,7 & 20 & 79.08 & 48,9 to 100 & 13,6 & $<0,0001$ \\
\hline Problem score & 50.38 & 6,7 to 91,3 & 21,7 & 22.54 & 2,1 to 91,3 & 22,9 & 0,0001 \\
\hline Global score & 35.77 & 0 to 58,3 & 21,2 & 74.45 & 50 to 100 & 15,3 & $<0,0001$ \\
\hline
\end{tabular}

Table 2: Shows the results of both questionnaires applied before $\mathrm{S} 1$ and five years after S2. Seventeen patients were included in both questionnaires ( $\mathrm{n}=$ Baseline, $\mathrm{SD}=$ Standard Deviation).

Spearman correlation was used when analyzing short and long stumps and found no statistically significant differences between different stump lengths regarding SF-36 and Q-TFA subscores.

Prosthetic survival was analyzed in a Kaplan-Meier graph (Figure 1), showing an $81 \%$ survival rate at 5 -year.

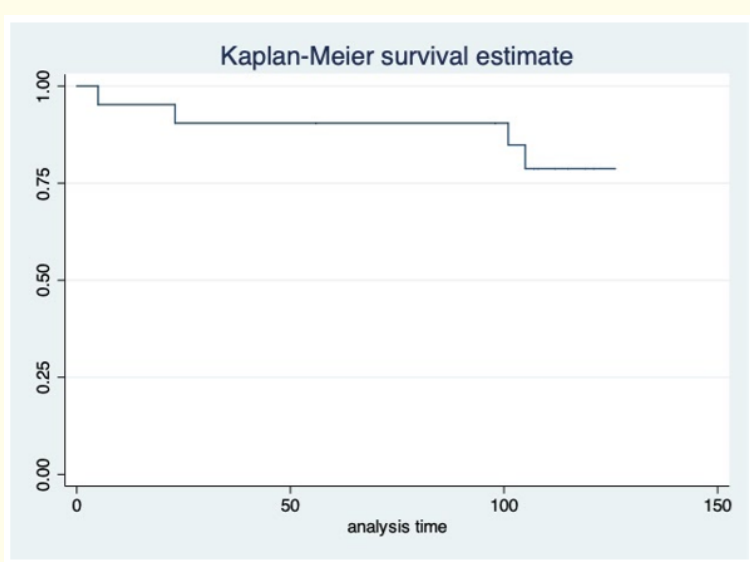

Figure 1: Survival rate at 5-year. Kaplan-Meier.
Our objective was to review functional outcome, implant survival, and complications in patients with transfemoral amputations and osseointegration. This novel technique has proven favorable functional outcomes, evaluated with specific surveys for transfemoral amputees (Q-TFA) and general quality of life surveys (SF-36); both scores are validated for amputees' outcome evaluation [15].

The first case series reported by Branemark highlighted complications and survival of this procedure [16]. Later, the same author published a prospective study, with the surveys mentioned above. In this previous study, the mean survival at two years followup was 92\%, showing an improvement in all items evaluated with Q-TFA, especially in the general situation, improving in 69\% cases. In the specific item analysis, all four items showed a significant improvement [17].

In our analysis, all four items of the Q-TFA survey showed a significant improvement.

The use score subsection considers the daily amount of time of prosthesis use, using 15 hours as maximum, so the closer to 100 , the longer the prosthesis is used. As Thomson., et al. report in their series, prosthesis use is high, with $82 \%$ to $90 \%$ of patients reporting daily use [3]. 
The mobility score evaluates the ability to change and maintain different postures with three questions. These refer to the conditions on which the patient can wander around: the need for walking aids, walking habits (frequency and length of the walk), and capability (the ability to perform locomotor activities without considering the difficulties). A higher score means more use of the prosthesis in different situations and less need for walking aids. The overwhelming majority of amputees who change from a traditional socket prosthesis to an osseointegrated prosthesis improve dramatically, both subjectively and objectively. One study showed that when amputees changed from a socket prosthesis to an osseointegrated prosthesis, improvements on the Q-TFA (from 45.27 to 84.86 points), Short Form-36 Physical Component Summary (from 36.97 to 49.00 points), 6 Minute Walk Test (from 286.25 to 512.72 meters) and the Timed Up and Go test (from 13.86 to 9.12 seconds), improvements were consistent noted [18].

Problems score to evaluate the presence or absence of problems, difficulties, or complications related to the amputation, prosthesis, and its use. The same questions are answered again, referring to the impact on each of the previously evaluated items' quality of life. A higher score means more problems related to the questioned items.

The global score sums up the patient's perception regarding the prosthesis in three questions evaluating the level of function, problems related to prosthesis use, and general situation as an amputee. The higher the score, the better the global perception of the patient regarding his condition. Improved patient experience with the use of an osseointegrated prosthesis was related to osseoperception. This phenomenon is defined as the mechanical stimulation of a bone-anchored prosthesis transduced by mechanoreceptors likely located in the muscles, joints, skin, and other bone-adjacent tissues. The transduced signals travel to the central nervous system to cause passive awareness of the patient's sensorimotor position and function [19].

The SF-36 survey was designed to evaluate the quality of life in patients and the general population. Its main advantage is that it allows a correlating quality of life with health, evaluating different treatments. In the same fashion as Q-TFA, a statistically significant improvement was seen in all SF-36's items.

In our series of patients with a post-traumatic transfemoral amputation, subject to a worker's compensation insurance, the study subjects show high physical demands on their limbs, shown on the high percentage of secondary procedures abutment exchange due to its breakage. This complication has previously been reported, with a prevalence of $27-45 \%$ for intramedullary screw implants and $0-31 \%$ for press-fit implants [20]. Abutment breakage must be considered at the moment of the procedure indication, with particular attention to implant type and modification of activities or physical demands sustained by the extremity to extend the implant survival. Branemark correlated abutment mechanical complications with Q-TFA's mobility score and the better results were seen in patients with a higher prevalence of these complications [21]. Likewise, Hagberg., et al. recently published their results on osseointegrated femoral prosthesis with long-term follow-up (111 patients and 15-year follow up). The mean age was 45 years, and $70 \%$ were male, similar to our case series. They showed a fixture survival rate of $89 \%$ and $72 \%$ at 7 and 15 years and Q-TFA significantly better than preoperatively. They also showed high mechanical complications (55\%), with a positive correlation between abutment mechanical failure and higher activity levels, thus supporting our results [22].

Improvement in subscores, mainly prosthetic use and mobility, are similar to what Leijendekkers showed in his one-year followup transfemoral and transtibial osseointegrated case series. They stated that almost $98 \%$ of the enrolled patients would likely go through the osseointegration procedure again [10].

In our case series, patients with a secondary procedure due to abutment mechanical failure had significantly higher use and mobility Q-TFA scores than those without mechanical failure ( $p 0,01$ ). This could be explained by the higher use given by the first group of patients to their osseointegrated prosthesis.

Reetz and cols presented 39 patients with a five-year follow-up, reporting all the infectious events and classifying them into four types (types 1 and 2 were superficial as 3 and 4 were deep infections). Their results showed that $77 \%$ of the patients had at least one infectious episode with a total of 156 episodes. 148 of those (95\%) were type 1 or 2 (95\%). Eight episodes (5\%) were grade 3 , and those occurred in four patients. No septic loosening was reported. They also showed a prosthesis use subscore improvement from 71 to 100 ( $p<0,001)$, as well as global score improved from 33 to $75(\mathrm{p}<0.001)$ [23].

Nine surgical debridements secondary to infectious episodes were performed in our patients, and two implant removals secondary to deep infection. This is a higher rate than the average pre- 
sented in the literature, where infection requiring an additional surgical procedure occurs in only $5 \%$ to $8 \%$ of patients $[24,25]$.

In 2019, Branemark 2019 presented a 5-year follow-up prospective cohort. 55 limbs in 51 patients treated with transfemoral osseointegrated prosthesis were included. Their results highlighted a 5 -year survival rate of $95 \%$, with $45 \%$ of revision free survival rate at 5-years (other causes included, such as abutment failure). They also evaluated infectious and mechanical complications, as well as patient-reported outcomes (PRO). They conclude that osseointegrated patients improve in PRO, but at 5-years, deep infection and loosening risk increase [14].

We also found no differences between long and short stumps regarding subjective scores and abutment failures. We interpret this as length should not matter when it comes to favorable functional outcomes in osseointegrated patients. It can be explained that the limb length is somehow recovered with these prostheses (given its modularity), and limb mechanics and proprioception have improved.

Symptomatic neuroma requiring an intervention had a $22 \%$ prevalence. This number has not been addressed in previous reports; however, it is crucial to consider for the surgical technique, as it corresponds to a cause of persistent pain.

Our first experience results follow the tendency of what Branemark., et al. have shown previously, laying a benchmark for Latin America, as this is the first report in this region.

\section{Conclusion}

Traditional prostheses for post-traumatic transfemoral amputees have proven not free of complications. It was until 1990 with Dr Branemark's work that osseointegration for transfemoral amputation was approved. Patient reported outcomes show significant improvements especially regarding to limb function. A high survival rate has previously been reported and the most frequent reintervention in this case series is abutment revision surgery, mainly due to fatigue of the component. This phenomenon, in the light of our patients' results imply a higher use of the extremity and therefore it's shorter durability. The outcomes reported in this article highlight the good results of this novel technique, making it acceptable as well as a safe alternative for transfemoral amputees.

\section{Acknowledgements}

To Doctors Rainhold Garcia and Carlos Sandoval, the first developers of this technique in our institution.

\section{Conflict of Interest}

On behalf of all authors, the corresponding author states that there is no conflict of interest.

\section{Bibliography}

1. Allyn G., et al. "Ability of a wash regimen to remove biofilm from the exposed surface of materials used in osseointegrated implants". Journal of Orthopaedic Research 37.1 (2018): 248257.

2. Dudek NL., et al. "Dermatologic conditions associated with use of a lower-extremity prosthesis". Archives of Physical Medicine and Rehabilitation 86.4 (2005): 659-663.

3. Thomson S., et al. "Proximal Bone Remodeling in Lower Limb Amputees Reconstructed with an Osseointegrated Prosthesis". Journal of Orthopaedic Research 37.12 (2019): 2524-2530.

4. Andrango Castro EM., et al. "Identification of the critical level of implantation of an osseointegrated prosthesis for aboveknee amputees". Computer Methods in Biomechanics and Biomedical Engineering 20.14 (2017): 1494-1501.

5. Nicholas JJ., et al. "Problems Experienced and Perceived by Prosthetic Patients". JPO: Journal of Prosthetics and Orthotics 5.1 (1993): 36-39.

6. Gallagher P and Maclachlan M. "Adjustment to an artificial limb: a qualitative perspective". Journal of Health Psychology 6.1 (2001): 85-100.

7. Brånemark PI., et al. "Osseointegrated implants in the treatment of the edentulous jaw. Experience from a 10-year period". Scandinavian Journal of Plastic and Reconstructive Surgery 16 (1977): 1-132.

8. Adell R., et al. "Long-term follow-up study of osseointegrated implants in the treatment of totally edentulous jaws". The International Journal of Oral and Maxillofacial Implants 5.4 (1990): 347-359.

9. Hansson E., et al. "Patients with unilateral transfemoral amputation treated with a percutaneous osseointegrated prosthesis: a cost-effectiveness analysis". Bone and Joint Surgery 100B.4 (2018): 527-534.

10. Atallah R., et al. "Complications of bone-anchored prostheses for individuals with an extremity amputation: A systematic review. McKinley TO, ed". PLOS ONE 13.8 (2018): e0201821.

11. Sullivan J., et al. "Rehabilitation of the trans-femoral amputee with an osseointegrated prosthesis: the United Kingdom experience". Prosthetics and Orthotics International 27.2 (2003): 114-120. 
12. Örgel M., et al. "Perioperative mortality secondary to a pulmonary embolism during a surgical implantation of the EndoExo-Prosthesis: a case report". ESC Heart Failure 6.5 (2019): 1092-1095.

13. Hagberg K., et al. "Osseoperception and Osseointegrated Prosthetic Limbs". In: Gallagher P, Desmond D, MacLachlan M, eds. Psychoprosthetics. Springer London (2008): 131-140.

14. Brånemark RP., et al. "Osseointegrated Percutaneous Prosthetic System for the Treatment of Patients With Transfemoral Amputation: A Prospective Five-year Follow-up of Patient-reported Outcomes and Complications". Journal of the American Academy of Orthopaedic Surgeons 27.16 (2019): e743-e751.

15. Hagberg K., et al. "Questionnaire for Persons with a Transfemoral Amputation (Q-TFA): Initial validity and reliability of a new outcome measure". Journal of Rehabilitation Research and Development 41.5 (2004): 695.

16. Hagberg K and Brånemark R. "One hundred patients treated with osseointegrated transfemoral amputation prostheses-rehabilitation perspective". Journal of Rehabilitation Research and Development 46.3 (2009): 331-344.

17. Brånemark R., et al. "A novel osseointegrated percutaneous prosthetic system for the treatment of patients with transfemoral amputation: A prospective study of 51 patients". Bone and Joint Surgery 96-B.1 (2014): 106-113.

18. Haket LM., et al. "Periprosthetic cortical bone remodeling in patients with an osseointegrated leg prosthesis: BONE REMODELING IN OSSEOINTEGRATION". Journal of Orthopaedic Research 35.6 (2017): 1237-1241.

19. Lennerås M., et al. "The clinical, radiological, microbiological, and molecular profile of the skin-penetration site of transfemoral amputees treated with bone-anchored prostheses: TRANSFEMORAL AMPUTEES TREATED WITH BONE-ANCHORED PROSTHESES". Journal of Biomedical Materials Research 105.2 (2017): 578-589.

20. Prochor P and Sajewicz E. "A comparative analysis of internal bone remodelling concepts in a novel implant for direct skeletal attachment of limb prosthesis evaluation: A finite element analysis". Proceedings of the Institution of Mechanical Engineers 232.3 (2018): 289-298.

21. Stenlund P., et al. "Effect of load on the bone around boneanchored amputation prostheses: BONE-ANCHORED AMPUTATION PROSTHESES". Journal of Orthopaedic Research 35.5 (2017): 1113-1122.
22. Hagberg K., et al. "A 15-year follow-up of transfemoral amputees with bone-anchored transcutaneous prostheses: mechanical complications and patient-reported outcomes". Bone and Joint Surgery 102-B.1 (2020): 55-63.

23. Reetz D., et al. "Safety and Performance of Bone-Anchored Prostheses in Persons with a Transfemoral Amputation: A 5-Year Follow-up Study". Journal of Bone and Joint Surgery 102.15 (2020): 1329-1335.

24. Muderis MA., et al. "Two-Stage Osseointegrated Reconstruction of Post-traumatic Unilateral Transfemoral Amputees". Military Medicine 183.1 (2018): 496-502.

25. Matthews DJ., et al. "UK trial of the Osseointegrated Prosthesis for the Rehabilitation for Amputees: 1995-2018”. Prosthetics and Orthotics International 43.1 (2019): 112-122.

\section{Assets from publication with us}

- Prompt Acknowledgement after receiving the article

- Thorough Double blinded peer review

- Rapid Publication

- Issue of Publication Certificate

- High visibility of your Published work

Website: www.actascientific.com/

Submit Article: www.actascientific.com/submission.php

Email us: editor@actascientific.com

Contact us: +919182824667 